\title{
Combining Biophysical Modeling and Machine Learning to Predict Location of Atrial Ectopic Triggers
}

\author{
Eduardo J Godoy, Miguel Lozano, Ignacio Garcia-Fernandez, Rafael Sebastian \\ CoMMLab, Universitat de Valencia, Valencia, Spain
}

\begin{abstract}
The search for focal ectopic activity in the atria triggered from non-standard regions can be time consuming. The use of body surface potential maps to plan the intervention can be helpful, but require an advance processing of the data, that usually involves to solve an ill-posed inverse problem. In addition, changes in maps due to pathological substrate such as fibrosis might affect the expected electrical patterns. In this work, we use a machine learning approach to relate ectopic focus activity in different atrial regions with body surface potential maps, and consider the effects of fibrosis in various densities and distributions. Results show that as fibrosis increases over 15\% the systems has to increase the region size in which an ectopic focus should be searched, but keeps the performance over $90 \%$ when at least 64 electrodes are used.
\end{abstract}

\section{Introduction}

Focal atrial tachycardia (FAT) can be treated by radio frequency ablation with an acceptable long-term success rate. However, the localization of ectopic foci in the atria requires that an electrophysiologist maps the electrical activity on the endocardium to infer, based on his experience, the area where tissue should be ablated to eliminate the arrhythmia [1]. When electrical triggers are located in non-standard regions, or only generate a few ectopic activations, it can be complex and time consuming its localization [2]. In addition, the existence of fibrotic tissue makes the tissue prone to ectopic focus in non-standard locations, and complicates the already intricate patterns of activation.

The development of non-invasive techniques based on surface ECG data that can assist electrophysiologist intraoperatively to track the electrical triggers could improve radio-frequency ablation success rates. Atrial arrhythmic events induced artificially from an intracardiac catheter have been successfully related to indices derived from body surface potential maps (BSPM) [3]. Decision trees have also been proposed to help identify the source of FAT from BSPM data [4], although within a limited number of regions. In most of previous studies, the presence of fibrosis has been neglected, however, it is important to develop techniques for identification of FAT triggers that take into account the cardiac substrate. Ignoring the effects of fibrosis is a clear limitation of any method based on body surface potential maps, since current-resistance fibrotic barriers affect the activation patterns.

This study aims to show a methodology to estimate the location of ectopic foci by means of a machine learning system trained with multi-electrode ECG data generated by means of a detailed biophysical model of the atria and torso. The use of biophysical simulations allows the definition of hundreds of scenarios that are posteriorly run in a high-performance computer and do not require any further computation in the clinical environment [5]. The scenarios used to train the machine learning models include fibrosis in different proportions and distributions. A secondary goal is to assess how fibrosis affects the body surface potential maps and the detection of the ectopic sources.

\section{Material and Methods}

\subsection{Biophysical Modelling}

The biophysical simulations were carried out in a 3D model of the atria and torso previously developed [6,7], that considered the specific fibre orientation in 21 different anatomical regions, and tissue and cellular heterogeneity in 10 regions. The ionic cellular model used was Courtemanche for the healthy tissue and MacCannell for fibrotic tissue. A total of 475 simulations of $200 \mathrm{~ms}$ in 25 configurations of the atria (i.e., different distributions of fibrosis) were carried out (Figure 1, left panel). Simulations were performed solving the monodomain equation in the atrial domain (ELVIRA Solver), which were propagated to the torso surface solving an approximation of the bidomain equation. The model included five different distributions of fibrosis in 5 different densities, following the definition of four level of LA remodelling stage (quartile) of fibrosis associated to the ratio of fibrosis to atrial volume (Utah stage I: < 8:1\% (Q1); Utah Stage II: < 16\% (Q2); Utah Stage III: < $21 \%$ (Q3); Utah Stage IV: > 21\% (Q4)) [8]. That gave rise to 25 models, for which we simulate 18 ectopic focus plus sinus rhythm activation. As a result, we obtained body surface potential maps (BSPM) for each of the 475 
simulations performed (Figure 1, right panel).

All the models were stabilized in $3 \mathrm{D}$ using a train of 20 pulses from the sino-atrial node at a BCL of $500 \mathrm{~ms}$. Following FAT was stimulated with the same BCL from the different ectopic foci for each scenario.

\subsection{Machine Learning Pipeline}

The input for the machine learning pipeline consisted in the set of simulated body surface potential integral maps (BSPiM). Each BSPiM is obtained by calculating the integral of the P-wave signal at each of the 256 recording leads on the torso (see Figure 4, top row) [9]. First, BSPiM are clustered using a k-means algorithm, for $\mathrm{k}=[2,10]$ clusters/groups. The clustering is unsupervised and allows to check whether simulations close in the BSPiM space map to ectopic foci close in the atrial anatomy. Following, a support vector machine is trained with the BSPiM and the labels obtained from the clustering. Finally, a cross-validation is performed to analyse the accuracy of the method to predict the label associated to a BSPiM, which is related to the location of the corresponding ectopic focus on the atria (Figure 1, right panel). The whole procedure was completed for input BSPiMs with varying number of electrodes.

\section{Results}

Focal activity was triggered from 19 different atrial locations, and BSPiMs were collected using a number of electrodes that ranged from 2 to 256 (see Figure 4, top row). The density of fibrosis produced changes in the patterns of activation for a given ectopic focus that were very notorious for stage IV ( $>21 \%$ fibrosis). As can be seen in Figure 1, central column, the direction of the wavefront in the left atria free wall changes for the last two cases which are in IV stage. This was due to conduction blocks and delays in areas with dense patchy fibrosis. In some cases, the activation map and BSPiM showed completely different patterns compared to normal activation or activation with fibrosis with a ratio under $15 \%$.

From all BSPiM data, the ectopic foci were grouped in into atrial regions that vary between $\mathrm{K}=2$ and $\mathrm{K}=10$ regions. Figure 2 shows how the different BSPiM clusters map to the atria ectopic location when fibrosis was not present. The regions delimited by black lines over the atria define the source of an ectopic focus, and the colour is the group or cluster to which the corresponding BSPiM was assigned. As can be seen, neighbouring regions share the same colours, which means that all the BSPiM generated by these ectopic foci are mapped into a closed compact atrial region. The results from the crossvalidation showed that in general 32 electrodes were enough to predict the cluster to which an ectopic focus belongs with an accuracy of $90 \%$ in patient without presence of fibrosis or with a level of fibrosis of around $5 \%$. As the number of clusters increase, the coloured regions decrease in area (more precision in the prediction required), and it is more difficult to related BSPiM to cluster, however, the system still performed well using 64 electrodes (see Figure 4, scores M1r1).

As fibrosis increased, the system required at least 64 electrodes to reach the same accuracy. However, in cases with fibrosis over $15 \%$, the labels assigned to the atrial regions overlapped, which blurred the association between BSPiM and specific atrial regions, hampering its utility. This effect occurs when a given ectopic focus generates a BSPiM map that changes as we increase the fibrosis stage. In those cases, the SVM associates an ectopic focus to a region/cluster that differs from the expected one. As a result, one ectopic focus can map into many regions, and then the search area for a cluster increases and overlaps with the search area of different ectopic focus.

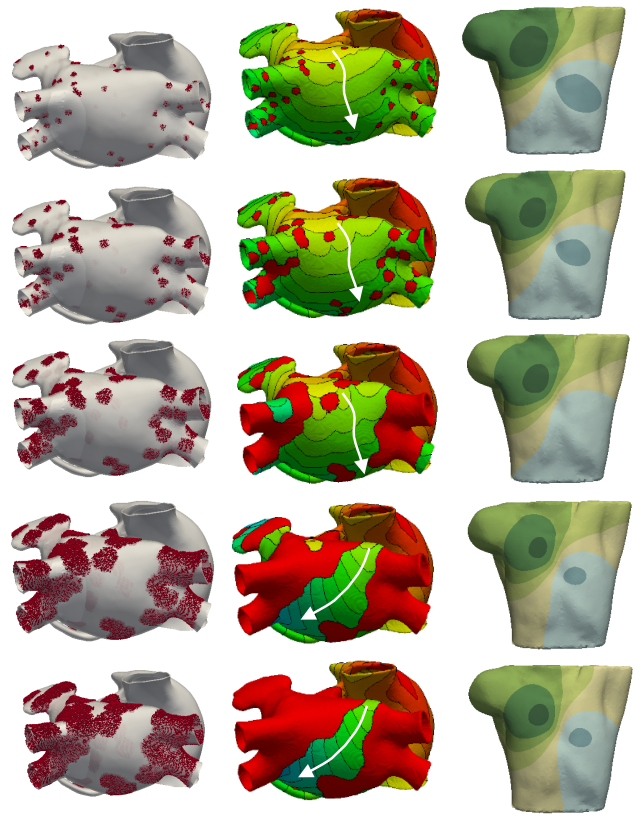

Figure 1: Atrial models including fibrosis (red areas), the local activation times in the atria, and the corresponding BSPiM in the torso for a given ectopic focus location.

As can be seen in Figure 3, for stages of fibrosis III and IV, a given patch which correspond to an ectopic focus is colored with several colors (dotted), meaning that it belongs to several clusters. This type of mapping, that is not one-to-one, hampers the localization of the ectopic focus.

However, if we allow the overlap, and train the SVM, the classification accuracy is still high as shown in Figure 4, Scores M3r1). 

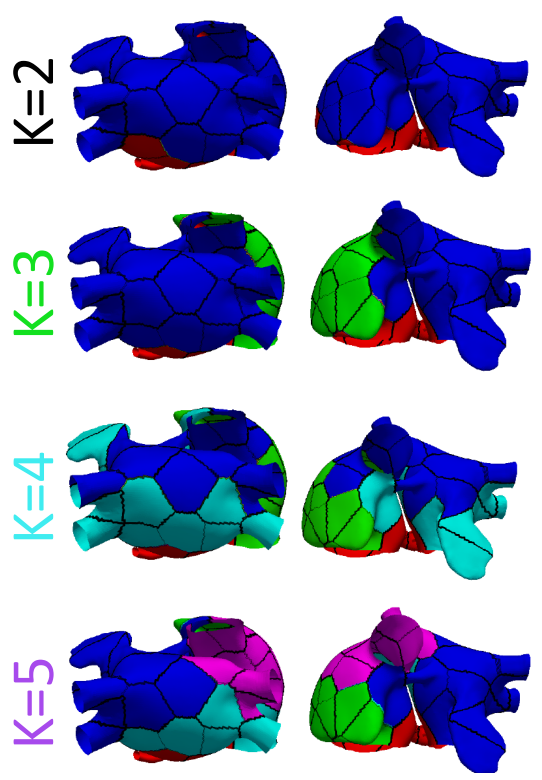

Figure 2: ectopic foci clustered in atrial regions (as a function of $\mathrm{K}$ chosen).
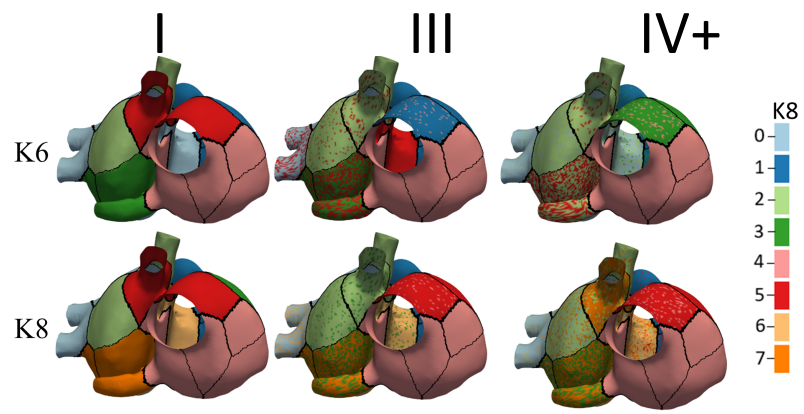

Figure 3: ectopic foci clustered in atrial regions for $\mathrm{K}=6$ and 8 clusters, and a ratio of fibrosis for stage I, III, and IV (upper limit). Colours depict the number of cluster in which a group of ectopic foci is map.

\section{Discussion and conclusions}

We have developed a machine learning pipeline that can be trained with biophysical model simulations to study the complexity of localizing ectopic focus from BSPM data. The procedure is similar to others based on BSPM and does not require the solution of an inverse problem to find the region where the ectopic focus is located. However, the presence of extensive dense fibrosis affects the detection, increasing the search area. There are no other studies that have analysed the effect of fibrosis distribution and density on the estimation accuracy of inverse algorithms.

The pipeline has been validated for healthy cases, although we do not have clinical data for patients showing fibrosis, and therefore results should be considered with caution. As future work, different atrial geometries and orientations within the torso should be considered to account on the patient to patient variability, which could affect the ECGs and BSPMs.
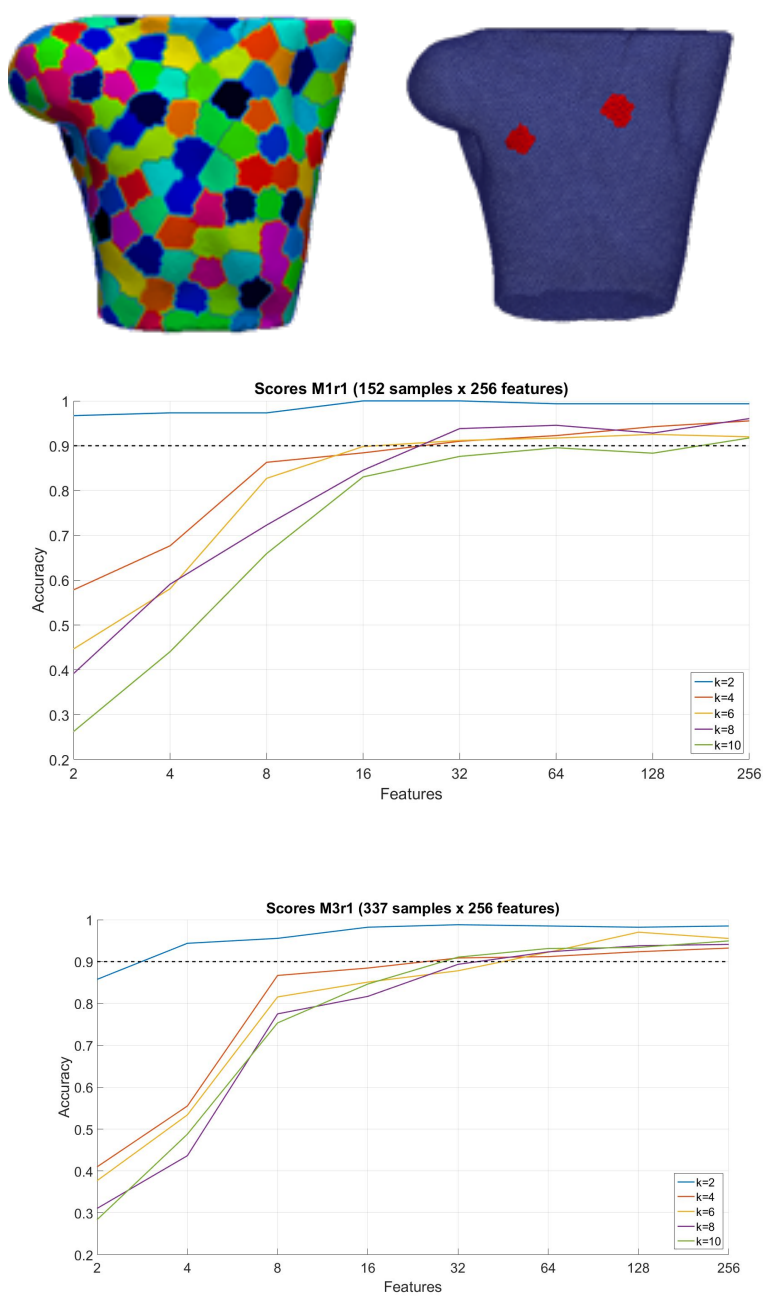

Figure 4: Location of the electrodes on the BSPM (max. 256) and the results for the cross-validation as a function of the number of electrodes and ectopic clusters.

\section{Acknowledgements}

This study is supported by the e3DTorso project (TIN2014-59932-JIN) from the Spanish Ministerio de Ciencia y Competitividad and FEDER funds. 


\section{References}

[1] P. Santangeli, F.E. Marchlinski. "Techniques for the provocation, localization, and ablation of non-pulmonary vein triggers for atrial fibrillation." Heart Rhythm vol. 14, pp. 1087 - 1096, 2017.

[2] P. Santangeli, E.S. Zado, M.D. Hutchinson, M.P. Riley, D. Lin, D.S. Frankel, et al. "Prevalenceand distribution of focal triggers in persistent and longstanding persistent atrial fibrillation." Heart Rhythm, vol. 13, pp. $374-382,2016$.

[3] A.J. Shah, M. Hocini, P. Pascale, L. Roten, Y. Komatsu, M. Daly, et al. "Body surface electrocardiographic mapping for non-invasive identification of arrhythmic sources." Arrhythmia \& electrophysiology review Vol. 2, pp. 16-22, 2013

[4] P.M. Kistler, K.C. Roberts-Thomson, H.M. Haqqani, S.P. Fynn, S. Singarayar, J.K. Vohra, et al. "P-Wave Morphology in Focal Atrial Tachycardia. Development of an Algorithm to Predict the Anatomic Site of Origin." Journal of the American College of Cardiology Vol. 48, pp. 1010-1017, 2006.

[5] A. Lopez-Perez, R. Sebastian J.M. Ferrero JM. "Three-dimensional cardiac computational modelling: methods, features and applications." Review Biomedical Engineering Online. Vol. 14(35), pp. 1 - 31, 2015.

[6] A. Ferrer-Albero, E.J. Godoy, M. Lozano, L. Martínez-mateu, F. Atienza, J. Saiz, R. Sebastian. "Noninvasive location of atrial ectopic beats by using Body Surface P-wave integral Maps." PLoS ONE Vol. 12(7):e0181263, pp. 1 - 23, 2017.

[7] A. Ferrer, R. Sebastian, D. Sanchez-Quintana, J.F. Rodriguez, E.J. Godoy, L. Martinez, J. Saiz. "Detailed anatomical and electrophysiological models of human atria and torso for the simulation of atrial activation." PLOS One, Vol. 10(11):e0141573, pp. 1 - 29, 2015.

[8] R.S. Oakes, T.J. Badger, E.G. Kholmovski, N. Akoum, N.S. Burgon, E.N. Fish, et al. "Detection and quantification of left atrial structural remodeling with delayed-enhancement magnetic resonance imaging in patients with atrial fibrillation." Circulation Vol. 119, pp. 1758-1767, 2009.

[9] A. SippensGroenewegen, F.X. Roithinger, H.A.P. Peeters, A.C. Linnenbank, N.M. Van Hemel, P.R. Steiner, et al. "Body surface mapping of atrial arrhythmias: Atlas of paced $\mathrm{P}$ wave integral maps to localize the focal origin of right atrial tachycardia." Journal of Electrocardiology. Vol. 31, pp. 85-91, 1998.
Address for correspondence:

Rafael Sebastian

Computational Multiscale Simulation Lab (CoMMLab), Universitat de Valencia. Escuela Técnica Superior de Ingenierías. Av. de la Universidad s/n, 46100 - Valencia (Spain).

rafael.sebastian@uv.es 